
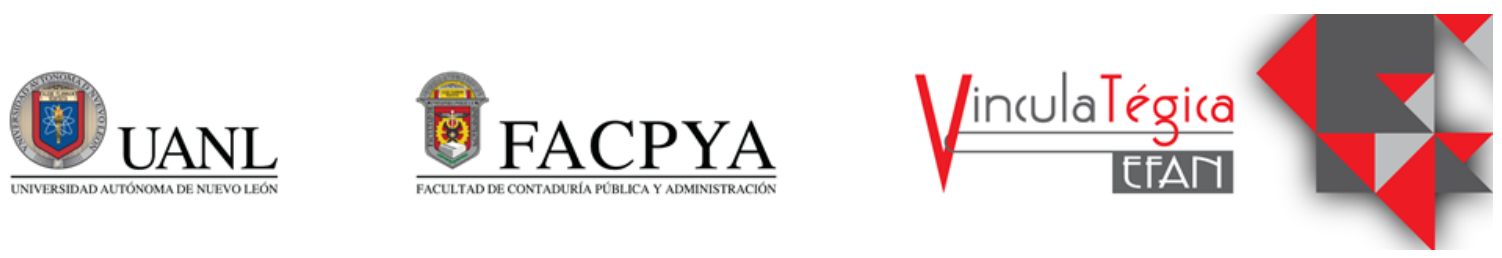

\title{
El uso de las Herramientas de Calidad en Micro y Pequeñas empresas de Altamira (Tamaulipas)
}

\author{
Nora Hilda González Durán ${ }^{1}$, María Elena Martínez García ${ }^{2}$ y Juan Antonio Olguín Murrieta ${ }^{3}$ \\ ${ }^{1}$ Universidad Autónoma de Tamaulipas, nhgonzale@docentes.uat.edu.mx, Centro Universitario Zona Sur de \\ Tamaulipas , 8331406135 . \\ ${ }^{2}$ Universidad Autónoma de Tamaulipas, memarti@docentes.uat.edu.mx, Centro Universitario Zona Sur de \\ Tamaulipas, 8335130270 \\ ${ }^{3}$ Universidad Autónoma de Tamaulipas, jaolguin@docentes.uat.edu.mx, ,Centro Universitario Zona Sur de \\ Tamaulipas, 8333229998
}

Información del artículo revisado por pares

Fecha de aceptación: junio-2021

Fecha de publicación en línea: diciembre-2021

DOI: https://doi.org/10.29105/vtga7.2-48

\section{RESUMEN}

La situación actual de nuestra economía no está siendo la ideal para el desarrollo de la micro y pequeñas empresas (MyPes) en México, las variables que componen el medio ambiente en el que se desenvuelven están siendo más crueles con su funcionamiento. Conocer las problemáticas a las que se enfrentan las MyPes, permitirá que, la toma de decisiones sea más apegada a la realidad y por consecuencia tener mayores posibilidades de éxito. La investigación se desarrolló en la ciudad de Altamira, Tamps.; con la finalidad de que los empresarios conozcan las características del entorno en el que se desenvuelven considerando entre otras el aspecto económico, el aspecto administrativo, y otros más que pudieran derivar en el cierre de empresas.

La investigación tiene como objetivo diseñar y proponer un Plan de Mejora Continua que pueda ser implantado a las MyPes establecidas en la Ciudad de Altamira, Tamps, que tenga como fundamento la información recabada directamente de las mismas en cuanto al uso de Herramientas de Calidad.

Para tal fin, los objetivos se enfocaron directamente en el uso y valoración de la Herramientas de Calidad en las MyPes situadas por conveniencia en la zona comercial del municipio de Altamira, Tamps. La muestra que se eligió fue no probabilística para este estudio, y por las condiciones ambientales prevalecientes se optó por un Muestreo por Conveniencia, apoyándose en el uso de una encuesta previamente diseñada y validada para tal efecto, como herramienta para la recopilación de datos.

Palabras Clave: Entorno, Herramientas de Calidad, Muestreo, Variables.

Código JEL: M21

\section{ABSTRACT}

The current situation of our economy is not being ideal for the development of micro and small companies (MyPes) in Mexico, the variables that make up the environment in which they operate are being more cruel with their operation. Knowing the problems that MSEs face will allow decision-making to be closer to reality and consequently have a greater chance of success.

The research was developed in the city of Altamira, Tamps .; In order for entrepreneurs to know the characteristics of the environment in which they operate, considering, among others, the economic aspect, the administrative aspect, and others that could lead to the closure of companies 
The objective of the research is to design and propose a Continuous Improvement Plan that can be implemented in the MyPes established in the City of Altamira, Tamps, based on the information collected directly from them regarding the use of Quality Tools.

To this end, the objectives were focused directly on the use and assessment of the Quality Tools in the MyPes located for convenience in the commercial area of the municipality of Altamira, Tamps. The sample that was chosen was non-probabilistic for this study, and due to the prevailing environmental conditions, a Convenience Sampling was

\section{1.- INTRODUCCIÓN}

En los albores de la humanidad el hombre tenía que ser autosuficiente para satisfacer sus necesidades. Ante esta difícil situación, cada individuo se fue dedicando a una actividad diferente según sus habilidades. Es aquí donde se inicia la división del trabajo, el comercio, el dinero y posteriormente surgen las primeras empresas en su forma rudimentaria. $\mathrm{La}$ globalización ha permitido que en el mundo se desarrollen empresas de distintos tamaños, capacidades y necesidades según el giro que se tenga. Actualmente, es cada vez más promovido la figura de la microempresa como forma de autoempleo. Existen institutos y corporaciones alrededor del mundo que enseñan, financian y promueven a micro empresas. Este tipo de entidades han proporcionado una de las mejores alternativas para la independencia económica ya que representan una gran oportunidad, a través de la cual los grupos en desventaja económica han podido iniciar y consolidarse por méritos propios.

Las micro empresas comparten los siguientes rasgos: Operan con escalas bajas de producción, utilizan tecnologías adaptadas, son de propiedad familiar y su financiamiento procede de fuentes propias (Carrasco, 2005). En México, la Ley para el Desarrollo de la Competitividad de la Micro, Pequeña y chosen, relying on the use of a survey previously designed and validated for this purpose, as a tool for data collection.

Keywords: Environment, Quality Tools, Sampling, Variables.

JEL code: M21

Mediana Empresa publicada en el 2002 en el Diario Oficial de la Federación, refiere que el tamaño de las empresas denominadas micro comprenden a aquellas de 1 a 10 trabajadores, para el sector industrial, comercio y servicios, lo que implicaba dejar fuera al sector agropecuario.

Cabe destacar que, el INEGI (Instituto Nacional de Estadística y Geografía) con financiamiento del INADEM (Instituto Nacional del Emprendedor) y de BANCOMEXT (Banco Nacional de Comercio Exterior) llevaron a cabo la Encuesta Nacional sobre Productividad y Competitividad de las Micro, Pequeñas y Medianas Empresas (ENAPROCE) 2015, la cual arroja que el $97.6 \%(3,952,422)$ son microempresas y concentran el $75.4 \%$ del personal ocupado total. Le siguen las empresas pequeñas, que son un $2 \%(79,367)$ y tienen el $13.5 \%$ del personal ocupado. Las medianas representan $0.4 \%$ $(16,754)$ de las unidades económicas y tienen poco más del $11 \%$ de los ocupados.

En el caso de las microempresas en México, el INEGI muestra que, mientras más personas ocupadas tenga la microempresa menos probabilidad tiene de muerte, lo cual le da más esperanza de vida. Lo anterior se puede comprobar numéricamente en la Tabla 1.1: 
Tabla 1.1.- Probabilidad de muerte al primer año y esperanza de vida al nacer

de las microempresas en México.

Personas ocupadas $\quad$ Probabilidad de muerte al primer año Esperanza de vida al nacer de vida (años futuros de vida)

\begin{tabular}{c|c|c|}
\hline $0-2$ & $\mathbf{0 . 3 8}$ & $\mathbf{6 . 9}$ \\
\hline $3-5$ & $\mathbf{0 . 3 2}$ & $\mathbf{8 . 4}$ \\
\hline $6-10$ & $\mathbf{0 . 1 7}$ & $\mathbf{1 5 . 0}$ \\
\hline
\end{tabular}

Fuente: (INEGI, Boletín de Prensa No. 087/15, 2015)

Sin embargo y de acuerdo a experiencias propias derivadas de la interacción en el sector empresarial tanto como empresario y como empleado, no necesariamente las empresas pertenecientes a este rubro en nuestro país no mantienen esta tendencia, por el contrario, a mayor número de empleados los gastos se disparan y la economía de las empresas se ven deteriorada, más aún si se presenta algún tipo de recesión como el que actualmente estamos viviendo en México, provocada en primer instancia por el gobierno federal y su errada política económica $\mathrm{y}$ en segunda instancia el comportamiento del medio ambiente, en este caso la situación de salud generada por la pandemia.

Aunado a lo anterior, los responsables de estas empresas no saben cómo superar esas dificultades (es decir, "cómo resolver los problemas"). A veces los problemas se resuelven de manera intuitiva, pero frecuentemente los problemas se hacen crónicos y limitan las posibilidades de éxito de la empresa. Consecuentemente, una empresa que tenga la capacidad de "resolver los problemas" adquirirá una ventaja competitiva sobre sus competidores.

Ante este panorama adverso, existen ciertas herramientas de calidad que, por su sencillez y

Así mismo, la Revista Venezolana de Gerencia en el artículo Calidad para la Competitividad en las micro, pequeñas y medianas empresas, de la Ciudad de México, Saavedra, et al, del año 2017, señala a la calidad como impulsor de la competitividad en las efectividad, son idóneas para ayudar a resolver los problemas del día a día, y de esta manera permiten que las micro y pequeñas empresas que las utilicen cuenten con información que les permita mejorar (mejora continua) los resultados de sus decisiones.

\section{2.- ENFOQUE TEORICO}

En la actualidad existen diversas investigaciones relacionadas con el uso de las herramientas de calidad en las microempresas a nivel nacional e internacional.

Uno de estos ejemplos está en el artículo de la Red Internacional de Investigadores en Competitividad Vol. 5 Num. 1 del año 2011 en el artículo El uso de las herramientas de calidad en empresas MYPIMES del sur de Tamaulipas, el Dr. Olguín Murrieta analiza un grupo de empresas de este sector del sur de Tamaulipas, para identificar qué herramientas usan y en qué grado lo usan, y de esta forma identificar áreas de oportunidad que a través de COPARMEX Tampico puedan ser subsanadas y volverse más competitivas

micro, pequeñas y medianas empresas (PYME), por esta razón el objetivo de este trabajo consiste en determinar la relación que existe entre estos dos factores en las PYME de la Ciudad de México. Los principales hallazgos muestran que la 
implementación de sistemas de calidad es incipiente, sin embargo, existe relación significativa entre la calidad y la competitividad.

\section{Contextualización de la Ciudad de Altamira.-}

La ciudad de Altamira fue fundada el 2 de mayo de 1749, se encuentra ubicada en la porción sureste del Estado de Tamaulipas y conforma junto con las ciudades de Tampico y Madero, la progresista región del sur de Tamaulipas, siendo esta región uno de los dos motores económicos del estado. El municipio de Altamira cuenta con una zona comercial creciente, con un corredor industrial pujante en el que se encuentran establecidas empresas de los distintos continentes y cuenta además con un Puerto de altura ubicado entre la cuarta y quinta posición del sistema portuario de México y reconocido a nivel mundial.

\section{Herramientas de Calidad.}

Las Herramientas de Calidad de Ishikawa son un conjunto de instrumentos que apoyan a la administración de una organización y que, en muchas ocasiones, son desconocidas para quien elige emprender. Las Herramientas de Calidad más conocidas son:

1.- Diagrama Ishikawa

2.- Diagrama de Flujo

3.- Gráfica de Control

4.- Histograma

5.- Diagrama de dispersión

6.- Diagrama de Pareto

7.- Hoja de Control

\section{METODOLOGIA}

El presente estudio se define como de enfoque cualitativo debido a sus características de enfoque no estandarizados, es decir, no se miden numéricamente debido a que a través de sus datos denominados cualitativos se obtienen descripciones detalladas de situaciones que posteriormente, conducen a la indagación de una manera subjetiva reconociendo sus tendencias personales (Hernández, et al, 2006). Además, posee la bondad de evaluar el desarrollo natural de las problemáticas en las microempresas sin manipulación en la realidad del contexto físico.

Así mismo el estudio realizado se define como experimental ya que, dentro del enfoque cualitativo se forman una serie de conjunto de prácticas interpretativas. Se adquiere un punto de vista desde el interior de la situación, aunque se mantiene una distancia como un observador externo. Se da una relación de empatía con los encuestados.

La metodología a utilizada para la investigación se basa principalmente en el análisis de una muestra basada en encuestas realizadas tanto a empresarios como a empleados de las micro y pequeñas empresas seleccionadas y que además lo permitan, complementada con la observación. El objetivo es detectar el uso de las Herramientas de Calidad en las microempresas situadas en la Ciudad de Altamira, Tamaulipas.

La muestra a utilizar en este estudio se considera no probabilística por conveniencia debido a que son especificaciones (microempresasubicadas formalmente en la zona centro de Altamira). Del mismo modo, se procede a considerar una población desconocida debido a que no hay un sistema de datos actualizados ni en las dependencias de los tres niveles ni cámaras que registren el número de microempresas instaladas formalmente en la zona centro del municipio actualmente. Es decir, que el tamaño muestral con los datos proporcionados es de 43 elementos para el instrumento de recopilación de datos. 


\section{4.- RESULTADOS}

A continuación, se describen los resultados de la aplicación del instrumento de recolección de datos en los 43 elementos correspondientes al tamaño muestral dado, de los cuales sólo 38 fueron contestados.

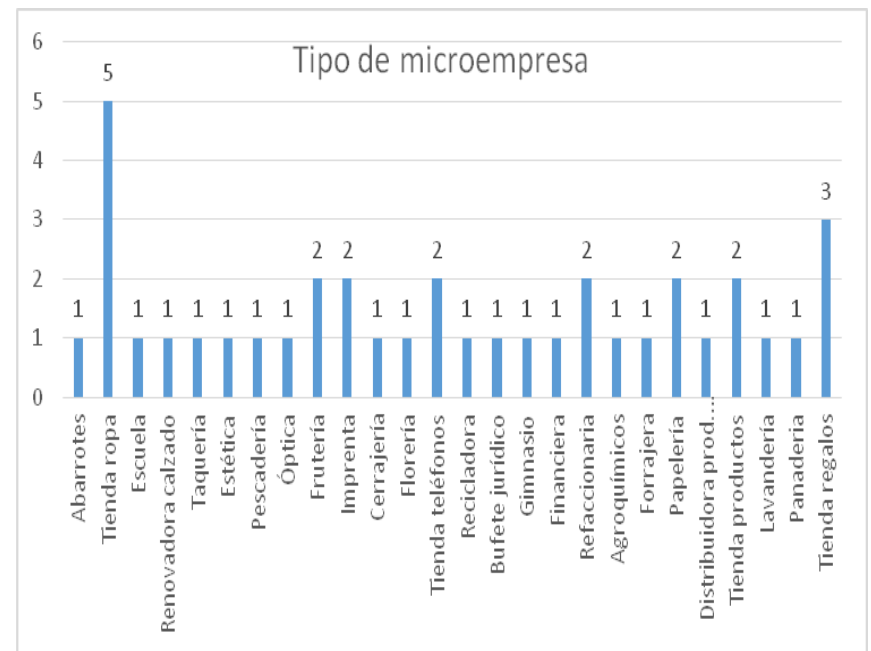

Tipo de microempresa y la cantidad que fueron encuestadas.(Fuente: Elaboración propia)

Cabe mencionar que las primeras 4 preguntas fueron redactadas para conocer aspectos propios de la microempresa, mientras que los restantes fueron enfocados a determinar los objetivos principales.

PAPEL DENTRO DEL NEGOCIO

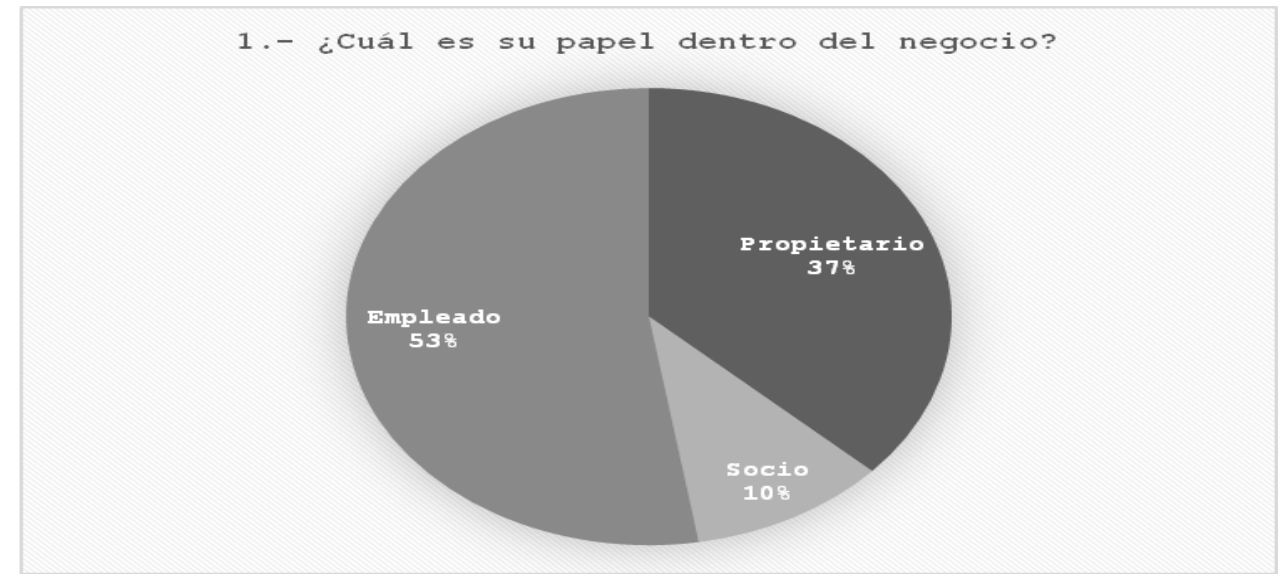

Fuente: Elaboración propia

Se puede apreciar que los encuestados fueron en su mayoría empleados (53\%) por lo que se deduce la poca participación activa del propietario.

\section{CATEGORIA EN LA EMPRESA}




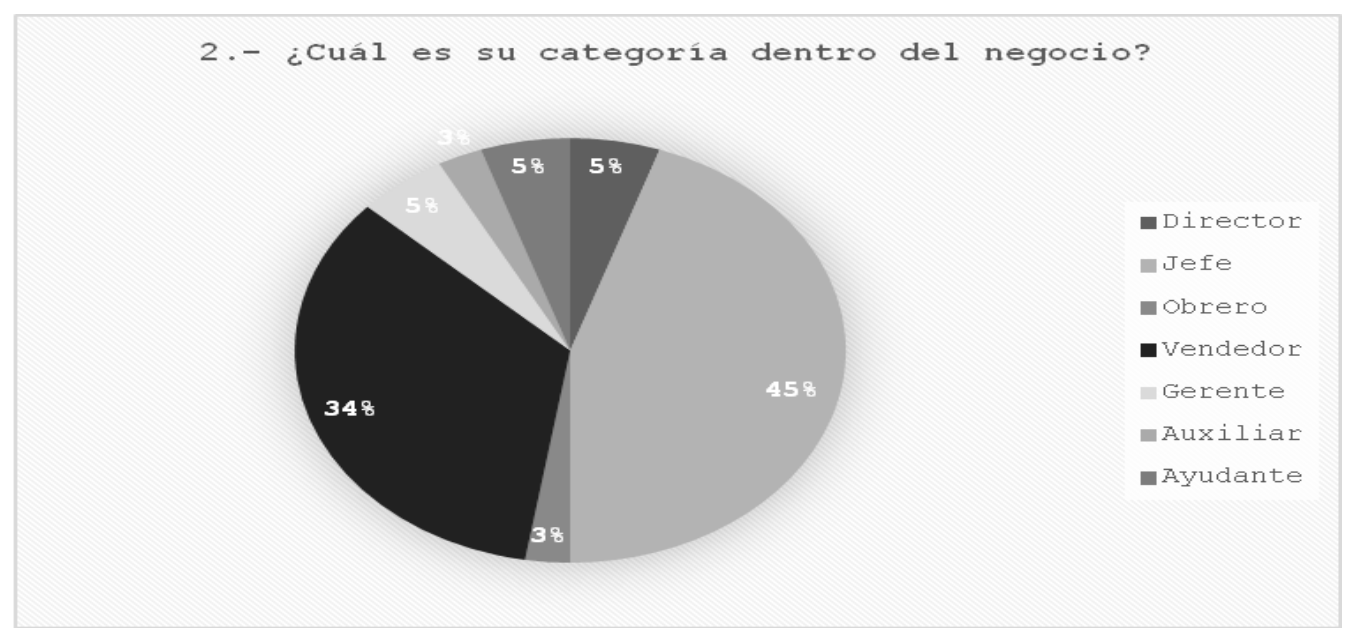

Fuente: Elaboración propia.

Se observa también que la mayor parte de los encuestados son jefes (45\%) por lo tanto se induce que las respuestas a la encuesta tendrán un alto grado de certeza.

NIVEL DE ESTUDIOS

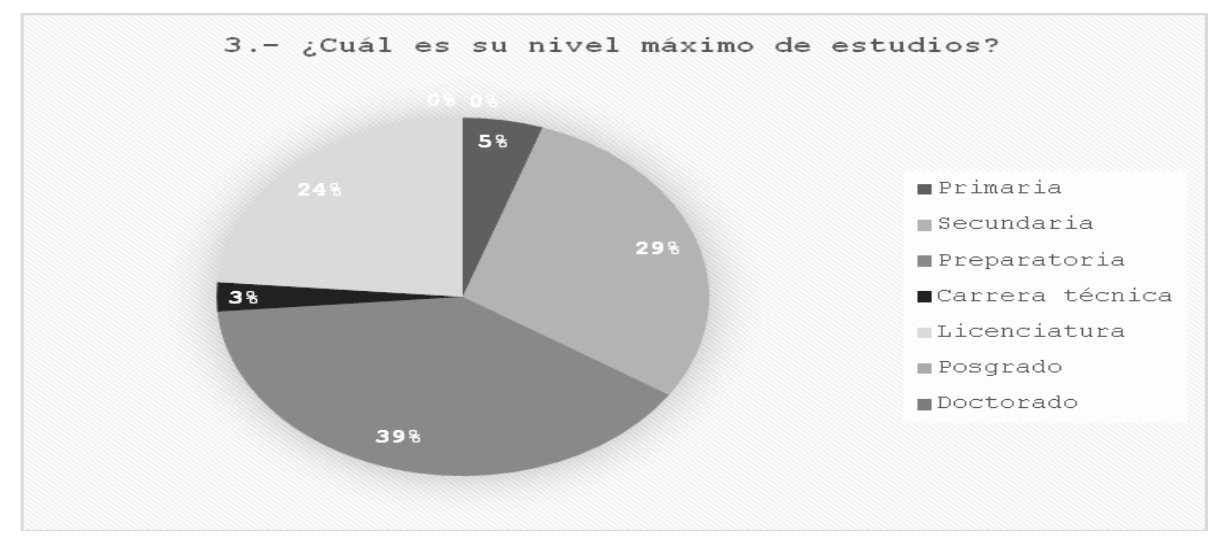

Fuente: Elaboración propia

El 39\% de los encuestados tienen niveles máximos de Preparatoria, el 24\% de los encuestados tiene una carrera profesional diferente siendo estas: Lic. En Ciencias de la Comunicación, Lic. En Administración de Empresas, Licenciado en Informática Administrativa, Lic. En Derecho, Lic. En Contaduría Pública, Lic. En Diseño Gráfico, Ing. En Electrónica, Ing. En Agronomía e Ing. Industrial.

Tomando en consideración los datos se observa que los propietarios y empleados son en su mayoría de nivel preparatoria por ser este un tipo de negocio que, por su tamaño y su metodología, no requieren una especialización, ya que al parecer con "sentido común" y un conocimiento básico del giro del negocio se puede llevar a cabo la administración. 


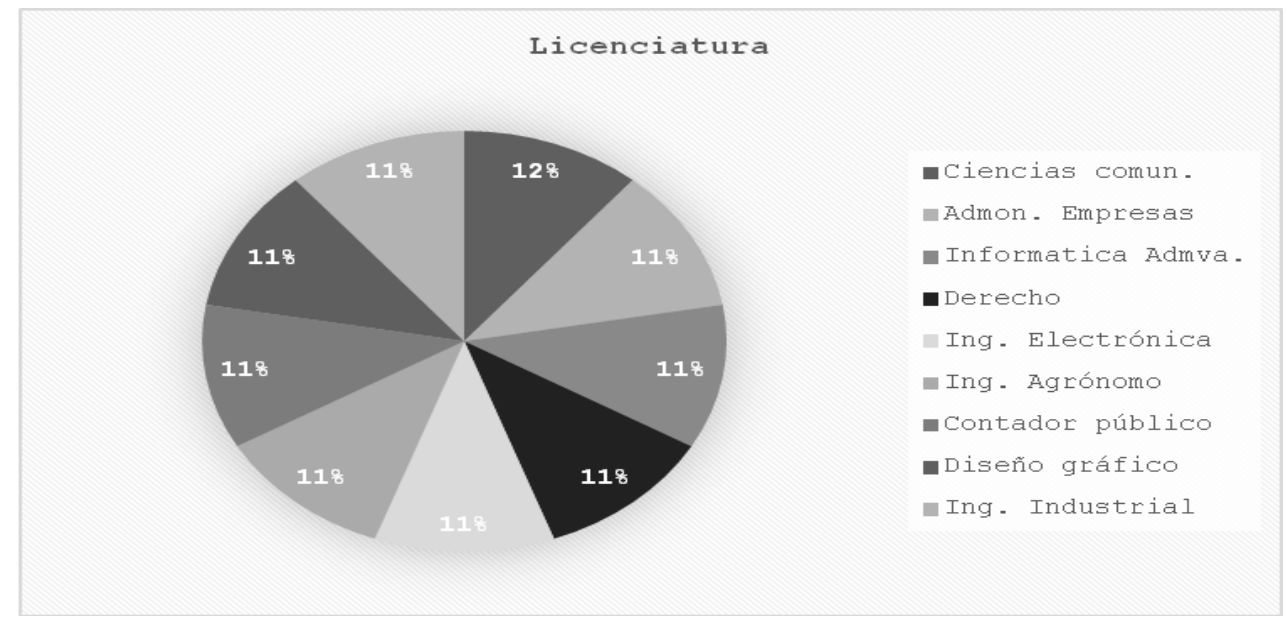

Fuente: Elaboración propia.

NUMEROS DE EMPLEADOS

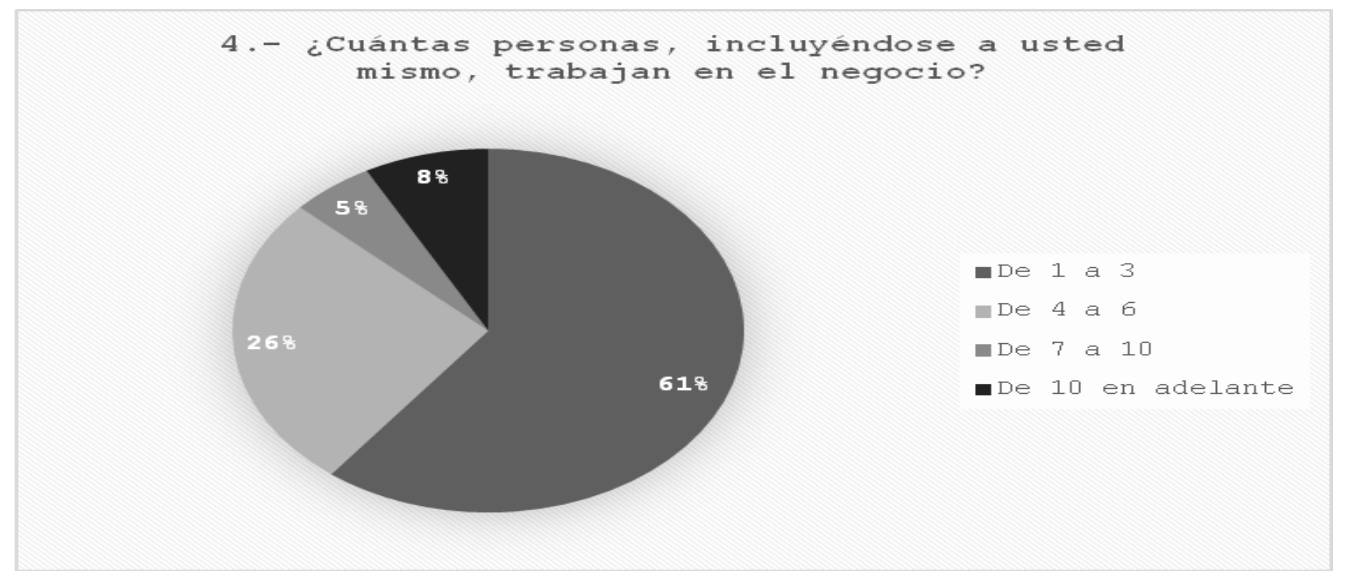

Fuente: Elaboración propia.

La presente pregunta arroja información con respecto al número de empleados en la microempresa. La información recabada es de vital importancia debido a que confirma la característica de la microempresa basada precisamente en el número de personas involucradas. El $61 \%$ de los negocios encuestados tienen una plantilla de 1 a 3 personas, mientras que solo el 5\% tiene una plantilla de 7 a 10 personas. Por lo tanto, sólo un $8 \%$ de las empresas encuestadas no pertenecen a la categoría de microempresas debido al número de personal.

HERRAMIENTAS UTILIZADAS 


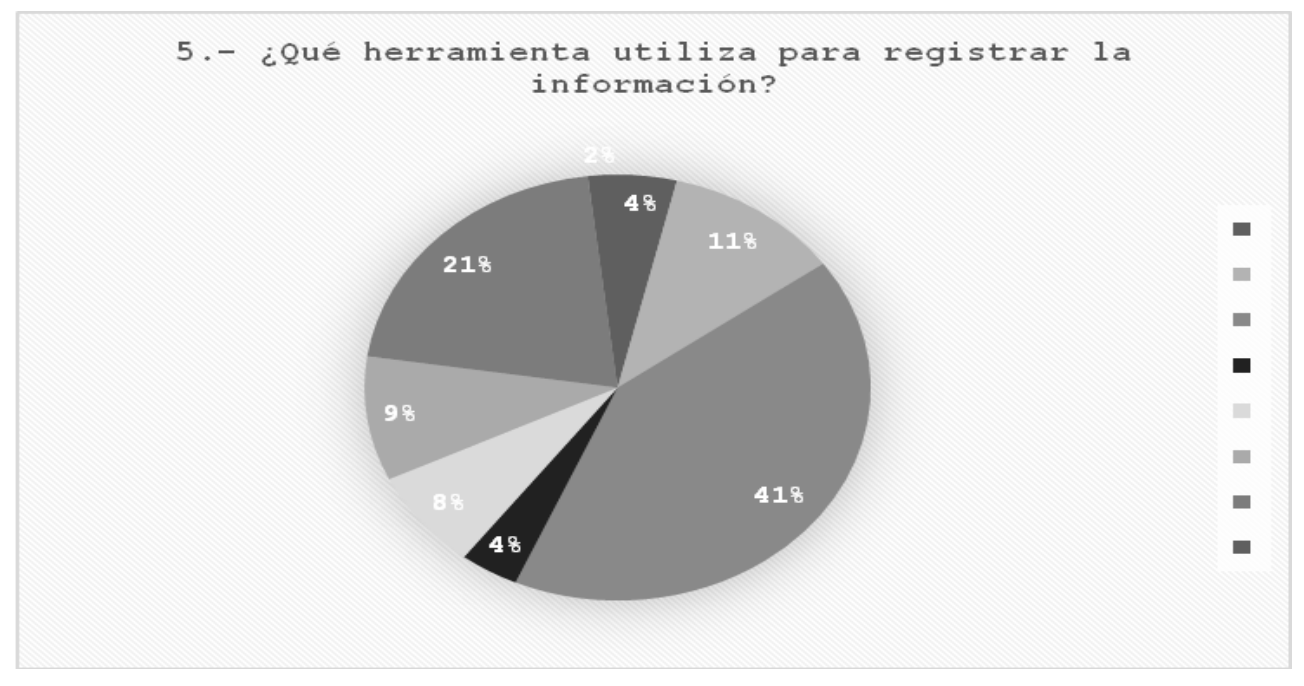

Fuente: Elaboración propia

En esta parte el encuestado identifica, mediante una descripción breve y entre las opciones presentadas, las herramientas utilizadas en la administración de la microempresa. El $41 \%$ dice utilizar la herramienta de la hoja de verificación o checklist en su negocio mientras que el 1\% utilizó la opción "Otros" para referirse a las notas de remisión como herramienta para recolectar información.

\section{REGISTRO DE INFORMACION}

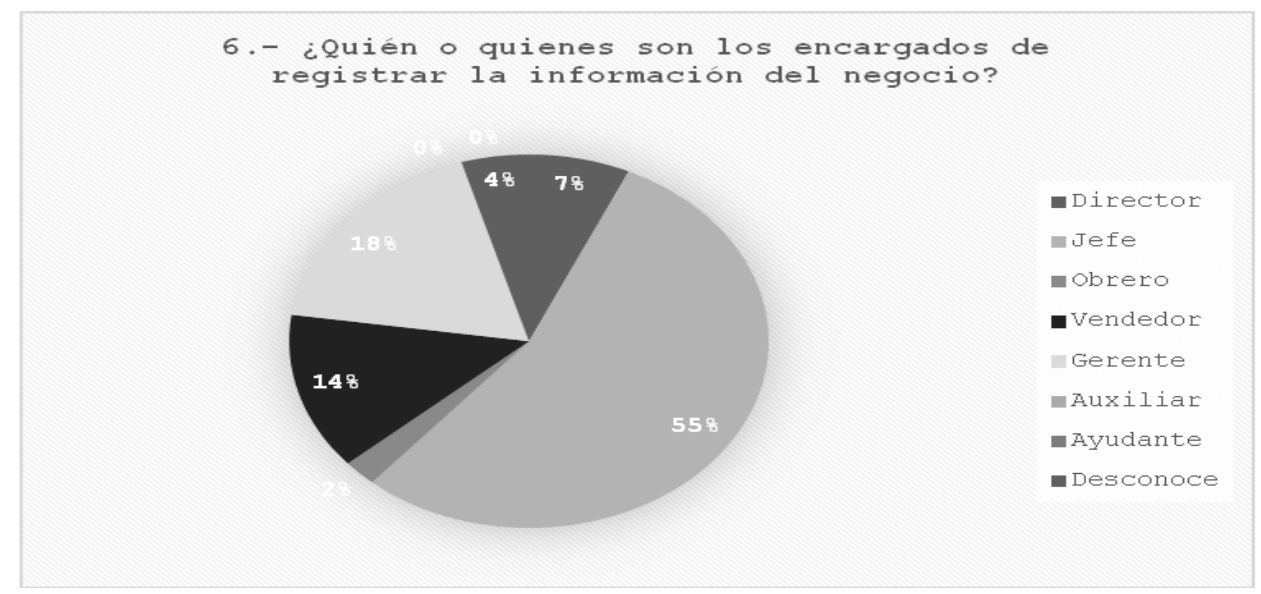

Fuente :Elaboración propia

Con la información obtenida, se pretende conocer quien maneja las herramientas de calidad como parte de sus responsabilidades, siendo el "Jefe" con un 55\% la persona dentro de la microempresa que se encarga de hacerlo, en contraste con la opción de "Obreros" que sólo obtuvo el 2\%.

\section{TIPO DE INFORMACION}




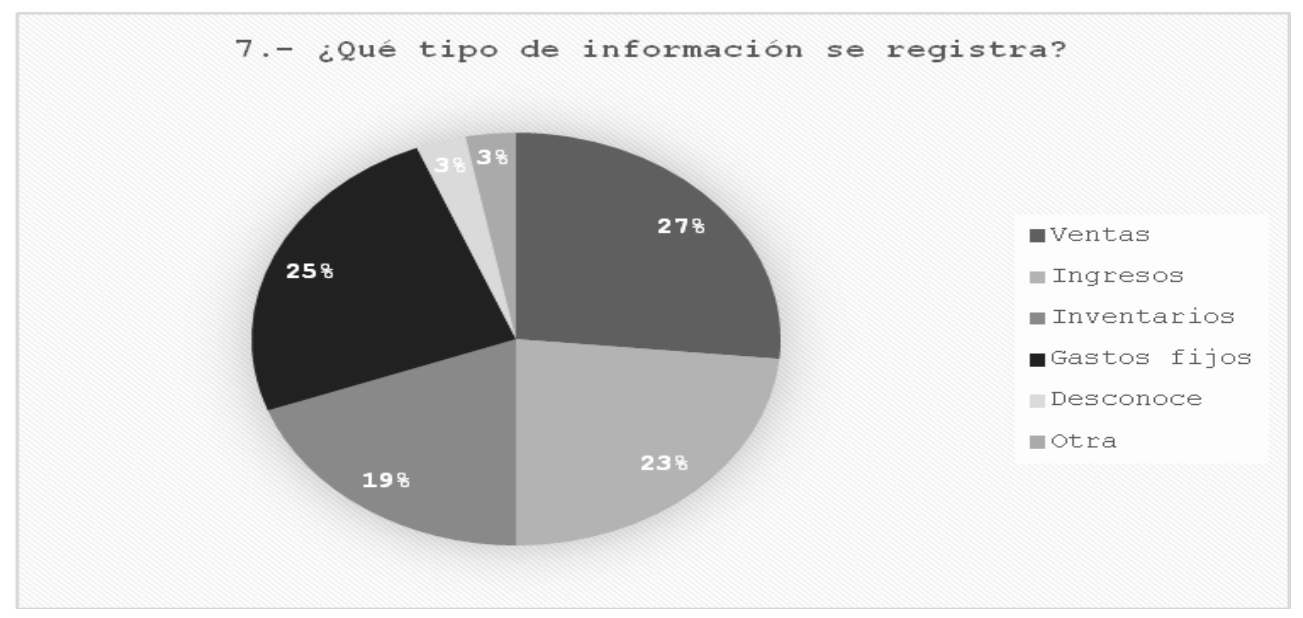

Fuente :Elaboración propia

El 27\% de los encuestados señalaron registrar las ventas mientras que 3\% señaló que desconocen o registran otra información distinta a la que se proporcionó en la encuesta. En cuanto a la opción de "Otra", los encuestados contestaron que registran otras opciones de información, siendo estas: Evaluaciones, investigación y archivo de juicios y expedientes, y cobranza.

\section{FORMA DE REGISTRO}

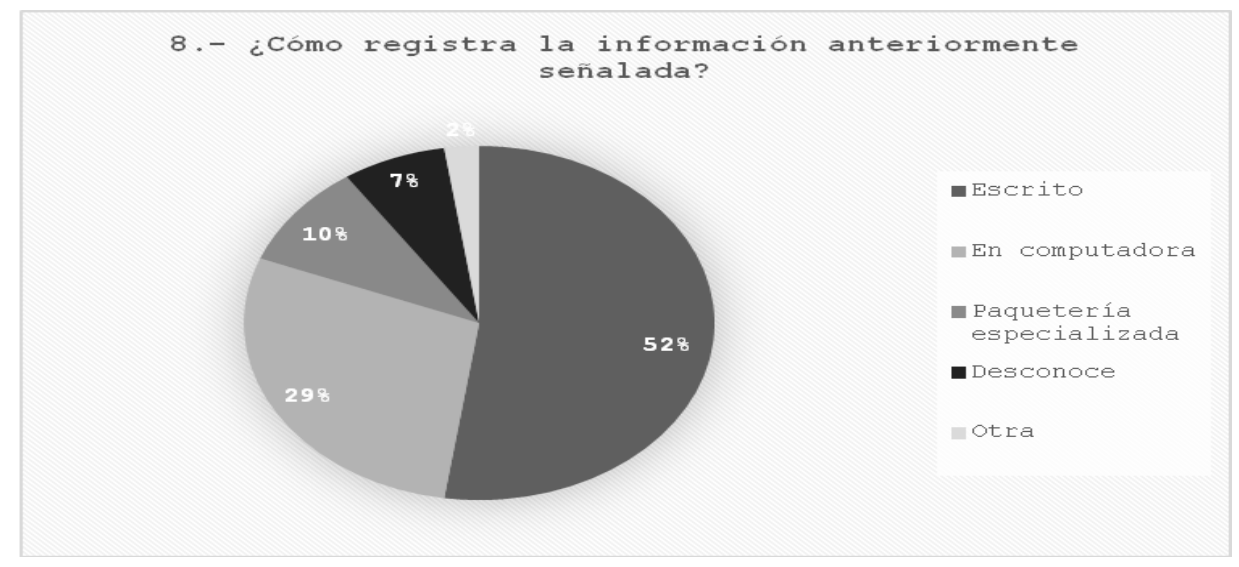

Fuente :Elaboranción Propia

Por medio de esta pregunta se busca conocer el medio por el cual se registra la información recabada por el encuestado. El 52\% de los encuestados comentan que registran su información por escrito en cuadernos, notas o formatos; mientras que la opción de "otro" con $2 \%$ se refiere a que el encuestado registra su información de manera mental. Se percibe a priori, por medio de las respuestas a esta pregunta que, nuevamente la preparación académica, así como "sentido común", tienen gran demanda al momento de registrar la información del negocio y no hacer uso de la tecnología.

\section{FRECUENCIA RECABADA}


9.- ¿Con qué frecuencia presenta la información recabada?

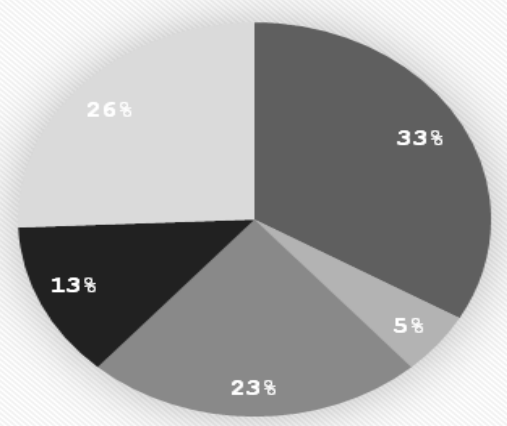

Fuente : Elaboración Propia

En esta gráfica se aprecia que el 33\% de los encuestados, es decir la mayoría, señalaron que presentan la información de manera diaria, mientras que el resto tiene un día o espacio en específico para poder recabar y presentar la información precisa.

\section{PRESENTACION DE LA INFORMACION}

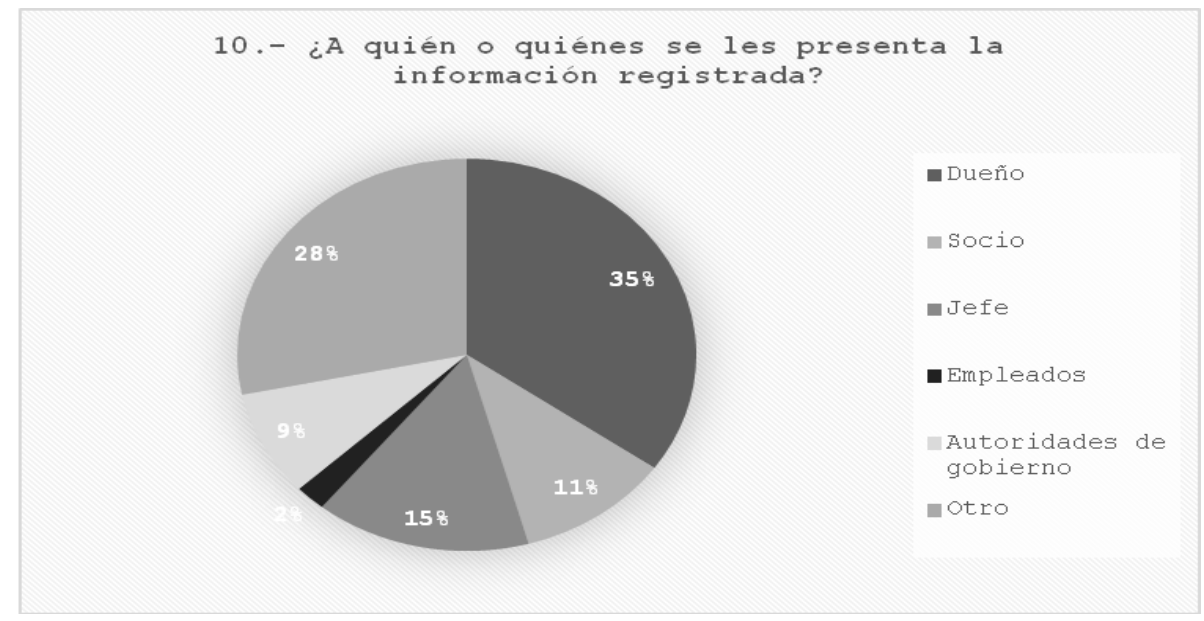

Fuente :Elaboración Propia.

Continuando con la línea de la pregunta anterior, la presente pregunta está hecha para conocer el destinatario de la información. El 35\% de los encuestados presentan la información a los dueños mientras que el 2\% de los encuestados les presentan la información a los mismos trabajadores. En cuanto a la opción de "Otro" el $28 \%$ de los encuestados tiene opciones diferentes siendo estas: Secretaría de Hacienda (2\%), Contador (17\%), Control interno (2\%) y desconoce $(7 \%)$.

\section{INFORMACION DEL NEGOCIO}




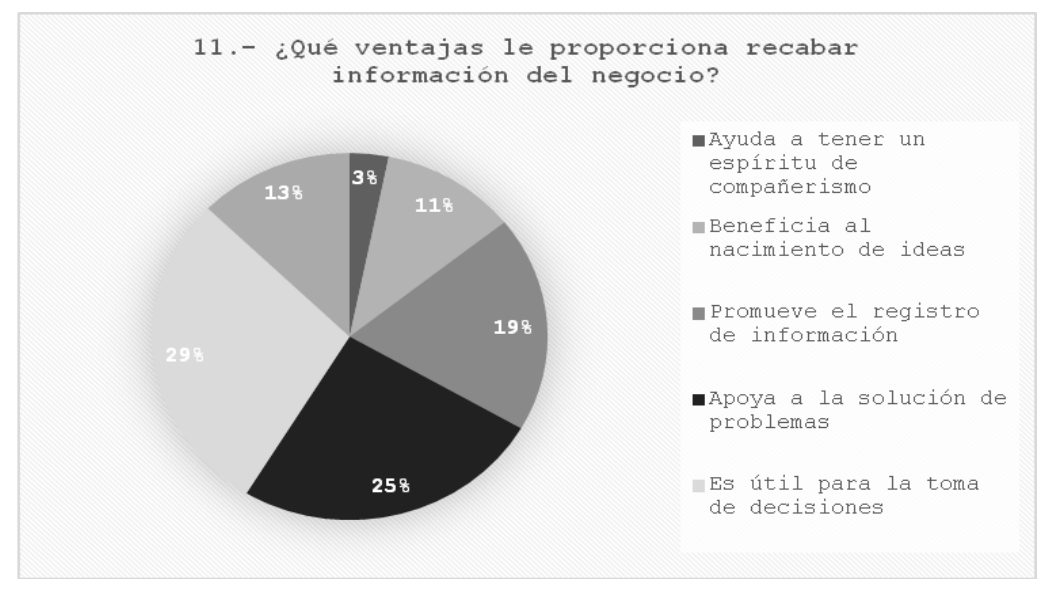

Fuente: Elaboración Propia

Con esta pregunta se busca saber de acuerdo con el criterio personal de cada encuestado el beneficio de tener información registrada mediante herramientas que permitan tener mayor control de la misma. El $29 \%$ de los encuestados consideran que la información que recaban es útil para la toma de decisiones mientras que el 3\% de los encuestados consideran que el recabar la información ayuda a fomentar un espíritu de compañerismo.

\section{5.- CONCLUSIONES}

Después de analizar la información en su conjunto se puede apreciar que la gran mayoría de los entrevistados no tiene conocimiento de las diferentes herramientas de calidad existentes y por lo tanto tampoco conoce los beneficios que pueden obtener de éstas, mucho menos las utilizan. Esto trae como consecuencia que las micro y pequeñas empresas ubicadas en la ciudad de Altamira Tamaulipas, no tengan conocimiento de información oportuna que les permita tomar mejores decisiones y, de esta forma contribuir al desarrollo de la empresa. Además de la mencionada información, se aprecia de manera general la forma de operar de cada una de las empresas, teniendo como resultado en su mayoría microempresas operadas por una a tres personas, con jefes contratados los cuales tienen un nivel académico de educación media superior (preparatoria). Lo anterior, lleva a deducir que quienes emprenden dan mayor importancia a cuidar el aspecto económico al contratar poco personal con conocimientos básicos, independientemente de su experiencia en el ramo. Se constató visualmente que en su mayoría son jóvenes los encargados de atender, siendo los varones los más comunes de ver atendiendo el negocio. En base a lo recabado se logró conocer que en la mayoría de las organizaciones es el "jefe" quién se encarga de recabar la información, y que los datos a recabar son mayormente los que se refieren a los ingresos (ventas). Se aprecia que la mayoría de los encuestados vacía la información al papel, es decir, que manejan un cuadernillo especial para apuntar la información relevante y que lo hacen con una frecuencia diaria. Otro punto relevante de la presente investigación es que, de acuerdo a la mayoría de los encuestados, la información se presenta a los dueños y en segundo lugar a los contadores o Hacienda para llevar a cabo las obligaciones fiscales. 


\section{REFERENCIAS BIBLIOGRAFICAS.}

Dr. Olguín Murrieta, J. A., M.E. Ing. Guerrero Rodríguez, J. d., \& Dr. Chavez Melendez, J. (2011). El Uso de Herramientas de Calidad en empresas MYPIMES del Sur de Tamaulipas. Red Internacional de Investigadores en Competitividad, 148- 166.

ENAPROCE- INEGI. (2015). Encuesta Nacional sobre Productividad y Competitividad de las Micro, Pequeñas y Medianas Empresas. Obtenido de http://www.inegi.org.mx/est/contenidos/proyectos/encuestas/establecimientos/otras/enaproce/defaul t_t.aspx

Escalante Vázquez, E. (2011). Análisis y mejoramiento de la calidad. México: Limusa.

Gob.mx . (2019). Enciclopedia de los Municipios y Delegaciones de México. Obtenido de http://siglo.inafed.gob.mx/enciclopedia/EMM28tamaulipas/municipios/28003a.html

Gobierno del Estado de Tamaulipas. (2016). Gobierno del Estado de Tamaulipas / Altamira. Obtenido de Gobierno de Altamira: http://www.tamaulipas.gob.mx/tamaulipas/altamira/

González Durán, N. H., Olguín Murrieta, J. A., Guzmán García, J. C., Guzmán Obando, J., \& Barragán Ramírez, R. (2017). Análisis De La Problemática En Ciudades Emergentes Del Sur De Tamaulipas. Palibro.

Guerra- López, I. (2007). Evaluación y Mejora Continua: Conceptos y Herramientas para la Medición y Mejora del Desempeño. Bloomington, Indiana: Global Business Press.

Hernández Sampieri, R., Fernández- Collado, C., \& Baptista Lucio, P. (2006). Metodología de la investigación. México, D.F.: McGraw- Hill Interamericana.

INEGI. (2018). DENUE Directorio Estadístico Nacional de Unidades Económicas. Obtenido de DENUE Directorio Estadístico Nacional de Unidades Económicas: http://www.beta.inegi.org.mx/app/mapa/denue/default.aspx\#

INEGI- ENAMIN . (2012). Encuesta Nacional de Micronegocios ENAMIN 2010. (INEGI, Editor) Recuperado el 01 de Febrero de 2018, de Encuesta Nacional de Micronegocios ENAMIN 2010. Documento metodológico: http://internet.contenidos.inegi.org.mx/contenidos/Productos/prod_serv/contenidos/espanol/bvinegi/ productos/metodologias/ENAMIN/enamin2010/ENAMIN2010.pdf

Maldonado Guzmán, G., Ojeda Hidalgo, J. F., Uc Heredia, L. J., Valdez Juárez, L. E., \& Medina Elizondo, M. (2017). La microempresa en México: un diagnóstico de su situación actual. México: Universidad Autónoma de Aguascalientes.

Molina Sánchez, R., López Salazar, A., \& Schimtt, C. (2016). Factores que influyen en los problemas del emprendimiento de las Mipymes: Una percepción de los expertos. Revista Internacional Administración y Finanzas, 95- 113. Obtenido de ftp://ftp.repec.org/opt/ReDIF/RePEc/ibf/riafin/riaf-v9n1-2016/RIAF-V9N1-2016.pdf\#page=97

Nava Carbellido, V. M. (2005). ¿Qué es la calidad?: Conceptos, gurpus y modelos fundamentales. México: Limusa. 
Organización de las Naciones Unidas. (2017). Naciones Unidas. Obtenido de https://www.un.org/es/events/smallbusinessday/

Pérez, A. (14 de Marzo de 2016). CEOLEVEL. Obtenido de Las siete herramientas básicas de la calidad: http://www.ceolevel.com/las-7-herramientas-basicas-de-la-calidad

Real Academia de la Lengua. (2018). Diccionario de la Real Academia Española. Obtenido de http://dle.rae.es/?id=6nVpk8P|6nXVL1Z

Ruiz- Falcó Rojas, A. (2009). Universidad Pontificia Comillas Madrid ICAI ICADE. Obtenido de Universidad Pontificia Comillas Madrid ICAI ICADE: https://s3.amazonaws.com/academia.edu.documents/35708455/herracalidad.pdf?AWSAccessKeyI $\mathrm{d}=$ AKIAIWOWYYGZ2Y53UL3A\&Expires $=1517894973 \&$ Signature $=u J t P 4 f s Q t V r R n 7 H r D Y B z 8$ YzIzPg\%3D\&response-contentdisposition=inline \%3B\%20filename\%3DHERRAMIENTAS_DE_CALIDAD.pdf

Saavedra García, M. L., Camarena Adame, M. E., \& Tapia Sánchez, B. (2017). Calidad para la competitividad en las micro, pequeñas y medianas empresas de la Ciudad de México. Revista Venezolana de Gerencia, 551.

Secretaría de Economía. (19 de 05 de 2017). Ley para el Desarrollo de la Competitividad de la Micro, Pequeña y Mediana Empresa. Diario Oficial de la Federación, pág. 2.

Sekhon, S., Singh Brar, G., \& Singh, S. (Agosto de 2014). A sigma approach to detect forging defects in small scale industry: A case study. International Journal of Engineering and Technical Research, 2(8), 33- 40. Recuperado el 04 de Marzo de 2018, de https://s3.amazonaws.com/academia.edu.documents/35056251/IJETR022220.pdf?AWSAccessKeyI $\mathrm{d}=$ AKIAIWOWYYGZ2Y53UL3A\&Expires $=1520320640 \&$ Signature $=\mathrm{kw} 9 \mathrm{qm} 53 \mathrm{CG} 8 \mathrm{Ucj} \% 2 \mathrm{FM} 3 \mathrm{x} 8$ M3O0G\%2FvMQ\%3D\&response-contentdisposition=inline\%3B\%20filename\%3DInternational_Journal_of_Engin

Suárez Barraza, M. F. (2007). El Kaizen: La Filosofía de la Mejora Continua e innovación Incremental detrás de la Administración por Calidad Total. México, D.F.: Panorama Editorial, S.A. de C.V. 